University of Nebraska - Lincoln

DigitalCommons@University of Nebraska - Lincoln

2-2-2003

\title{
X-Ray Radiation from Nonlinear Thomson Scattering of an Intense Femtosecond Laser on Relativistic Electrons in a Helium Plasma
}

K. Ta Phuoc

Laboratoire d'Optique Appliquée, Ecole Polytechnique, Chemin de la Hunière, France

A. Rousse

Laboratoire d'Optique Appliquée, Ecole Polytechnique, Chemin de la Hunière, France

M. Pittman

Laboratoire d'Optique Appliquée, Ecole Polytechnique, Chemin de la Hunière, France

J.P. Rousseau

Laboratoire d'Optique Appliquée, Ecole Polytechnique, Chemin de la Hunière, France

Victor Malka

Laboratoire d'Optique Appliquée, Ecole Polytechnique, Chemin de la Hunière, France, victor.malka@ensta.fr

See next page for additional authors

Follow this and additional works at: https://digitalcommons.unl.edu/physicsumstadter

Part of the Physics Commons

Phuoc, K. Ta; Rousse, A.; Pittman, M.; Rousseau, J.P.; Malka, Victor; Fritzler, S.; Umstadter, Donald P.; and Hulin, D., "X-Ray Radiation from Nonlinear Thomson Scattering of an Intense Femtosecond Laser on Relativistic Electrons in a Helium Plasma" (2003). Donald Umstadter Publications. 20.

https://digitalcommons.unl.edu/physicsumstadter/20

This Article is brought to you for free and open access by the Research Papers in Physics and Astronomy at DigitalCommons@University of Nebraska - Lincoln. It has been accepted for inclusion in Donald Umstadter Publications by an authorized administrator of DigitalCommons@University of Nebraska - Lincoln. 


\section{Authors}

K. Ta Phuoc, A. Rousse, M. Pittman, J.P. Rousseau, Victor Malka, S. Fritzler, Donald P. Umstadter, and D. Hulin

This article is available at DigitalCommons@University of Nebraska - Lincoln: https://digitalcommons.unl.edu/ physicsumstadter/20 


\title{
X-Ray Radiation from Nonlinear Thomson Scattering of an Intense Femtosecond Laser on Relativistic Electrons in a Helium Plasma
}

\author{
K. Ta Phuoc, ${ }^{1}$ A. Rousse, ${ }^{1, *}$ M. Pittman, ${ }^{1}$ J. P. Rousseau, ${ }^{1}$ V. Malka, ${ }^{1}$ S. Fritzler, ${ }^{1}$ D. Umstadter, ${ }^{2}$ and D. Hulin ${ }^{1}$ \\ ${ }^{1}$ Laboratoire d'Optique Appliquée, ENSTA, CNRS UMR7639, Ecole Polytechnique, Chemin de la Hunière, 91761 Palaiseau, France \\ ${ }^{2}$ Center for Ultrafast Optical Science, University of Michigan, 1006 Bonisteel Boulevard, Ann Arbor, Michigan 48109, USA
}

(Received 24 February 2003; published 5 November 2003)

\begin{abstract}
We have generated $\mathrm{x}$-ray radiation from the nonlinear Thomson scattering of a $30 \mathrm{fs} / 1.5 \mathrm{~J}$ laser beam on plasma electrons. A collimated $\mathrm{x}$-ray radiation with a broad continuous spectrum peaked at $0.15 \mathrm{keV}$ with a significant tail up to $2 \mathrm{keV}$ has been observed. These characteristics are found to depend strongly on the laser strength parameter $a_{0}$. This radiative process is dominant for $a_{0}$ greater than unity at which point the relativistic scattering of the laser light originates from $\mathrm{MeV}$ energy electrons inside the plasma.
\end{abstract}

DOI: 10.1103/PhysRevLett.91.195001

PACS numbers: 52.38.Ph, 52.25.Os, 52.50.Dg

The production of intense and ultrafast $\left(10^{-13} \mathrm{~s}\right)$ bursts of $\mathrm{x}$ rays from compact systems would have a dramatic impact in a number of scientific fields [1-3]. Laser-based experiments can provide ultrafast $\mathrm{x}$-uv $(20 \mathrm{~nm})$ and monochromatic $\mathrm{x}$-ray pulses $(0.5 \mathrm{~nm})$ from processes such as high harmonic generation from bound electrons in gases [4] and $K \alpha$ emission from solids [5,6]. The advent of high intensity laser systems now opens up the possibility to produce polychromatic hard $\mathrm{x}$-ray radiation while keeping the duration on the femtosecond time scale. It has been proposed to use the nonlinear motion of the relativistic electrons inside the laser field to produce high order harmonics of the incoming laser light [7-13]. This process is variously called nonlinear Thomson scattering as well as Larmor radiation. Such radiative emission was observed a few years ago by the detection of the second and the third harmonic light of a $1.053 \mu \mathrm{m}$ laser system [14] and extended to the 30th harmonic recently [15]. We report in this Letter on the radiation observed in the $\mathrm{x}$-ray spectral range, which was anticipated at relativistic quiver energies provided by the ultraintense laser fields. The results show the existence of several physical mechanisms for the $\mathrm{x}$-ray generation in this parameter regime.

Three main properties characterize the nonlinear Thomson scattering radiation [7-13]. First, the $\mathrm{x}$-ray intensity increases linearly with the electron number oscillating inside the laser field (indicative of an incoherent process). Second, the $\mathrm{x}$-ray spectrum is broad and peaked. Third, the emission is strongly anisotropic. Those unique properties can efficiently be used to distinguish the nonlinear Thomson scattering radiation from the other radiative processes during the laser-plasma interaction like the generation of high order harmonics from bound electrons, the isotropic Bremsstrahlung radiation, or radiative recombination of thermal electrons from the hot plasma. The spatial and spectral behaviors are fully governed by the experimental geometry and the trajectory of the electrons [16]. They are therefore directly related to the laser strength parameter $a_{0}=$ $e E / m_{0} \omega_{0} c=8.5 \times 10^{-10} \lambda_{\mu \mathrm{m}}\left(I_{\mathrm{W} / \mathrm{cm}^{2}}\right)^{1 / 2}$ (where $E$ is the amplitude of the laser field, $e$ is the electron charge, $m_{0}$ is the electron mass, $\omega_{0}$ is the laser frequency and $\lambda$ is the laser wavelength), the laser polarization, and the plasma parameters. We have performed test particle simulations to obtain the electron trajectory and all the characteristics of the radiation emitted as a function of the laser and plasma parameters. In this model the electron is submitted to a plane electromagnetic laser wave and to a one dimensional restoring force from the ions's background. The characteristics of the emitted radiation (power, spectrum, spatial distribution) are then obtained using the basic formulas of the radiation emitted by an accelerated charge [16]. If we consider the simplest case of a free electron oscillating inside the laser field at relativistic intensity $\left(a_{0}>1\right)$, the spatial distribution mainly consists of two lobes collimated in the forward direction for linearly polarized light with a comparatively weak backward contribution. The lobes are centered at an angle $\theta=$ $\tan ^{-1}\left(p_{\perp} / p_{x}\right) \sim 2 / a_{0}$ in the plane of the polarization with a width of $\sim 2 / a_{0}^{2} . p_{\perp}$ and $p_{x}$ are, respectively, the electron momentum perpendicular and parallel to the laser propagation axis. As $a_{0}$ is increased, the ponderomotive drift of the electron trajectory in the forward direction grows significantly and the spatial distribution becomes more and more collimated. For $a_{0}=5$, the characteristic angle of emission relative to the laser propagation axis is close to $23^{\circ}$ and the width of the lobes approaches $10^{\circ}$. For circular polarization, the trajectory is symmetric with respect to the laser axis and the emission pattern consists of a cone. The spectral distribution is broad and peaked at a maximum shifting to the shorter wavelength as $a_{0}$ is increased. It consists of harmonics lines with a spectral separation depending on $a_{0}$. In the present case of a very short driving laser pulse $(<100 \mathrm{fs})$, the oscillation motion of the electron is strongly modulated in the laser pulse envelop. The simulations have shown that the spectrum becomes continuous instead of consisting of discrete harmonic lines. A signal peaked at 
100 and $1000 \mathrm{eV}$ is foreseen for $a_{0}$ approaching 5 and 10 , respectively.

The experiment was conducted at the Laboratoire d'Optique Appliquée where we used a $30 \mathrm{fs}$ and $1.5 \mathrm{~J}$ (50 TW) Ti:Sa laser system. The experimental setup is shown in Fig. 1. The laser beam, $55 \mathrm{~mm}$ in diameter, was focused with an $f / 5.45$ off-axis parabolic mirror onto the front edge of a supersonic gas jet (3 $\mathrm{mm}$ in diameter) of helium and argon characterized from interferometric (Mach-Zender) measurements [17]. Electronic densities from $10^{18}$ to a few $10^{19} \mathrm{~cm}^{-3}$ were used in the experiment. The diameter of the focal spot was $6 \mu \mathrm{m}$ at full width at half maximum (FWHM) in intensity and contained $60 \%$ of the laser energy. The maximum incident laser intensity $(I)$ was $7 \times 10^{19} \mathrm{~W} / \mathrm{cm}^{2}$, which corresponds to a laser strength parameter $a_{0}=5.6$ for linear polarization. The x-ray radiation was collected with grazing incidence metallic mirrors (nickel or gold) over a solid angle of $1.5 \times 10^{-2} \mathrm{sr}$, and was focused onto a back illuminated $\mathrm{x}$-ray charge-coupled device (CCD) camera. Filters were positioned between the plasma and the detector to block the laser infrared light. A static and $0.5 \mathrm{~T}$ magnetic field was inserted between the plasma and the $\mathrm{x}$-ray spectrometer to deviate the electrons accelerated in the forward direction out of the detector. We selected spectral bands of the incoming $\mathrm{x}$ rays by combining the spectral absorption of the filters of different materials ( $\mathrm{Ti}, \mathrm{Zr}, \mathrm{Al}, \mathrm{Be})$ with thicknesses between $150 \mathrm{~nm}$ and $25 \mu \mathrm{m}$, and the spectral reflectivity of the mirrors. Our $\mathrm{x}$-ray spectrometer was sensitive to $\mathrm{x}$ rays in large spectral bandwidths from $20 \mathrm{eV}$ to $2 \mathrm{keV}$. The angular dependency of the $\mathrm{x}$-ray emission was obtained by rotating the entire spectrometer around the laser focal spot. A second arm of the laser system was used to optically probe, on each shot, the propagation of the beam inside the cylindrical gas jet. Electrons accelerated in the forward direction inside the plasma were characterized using radiochromic film and a set of filters in the

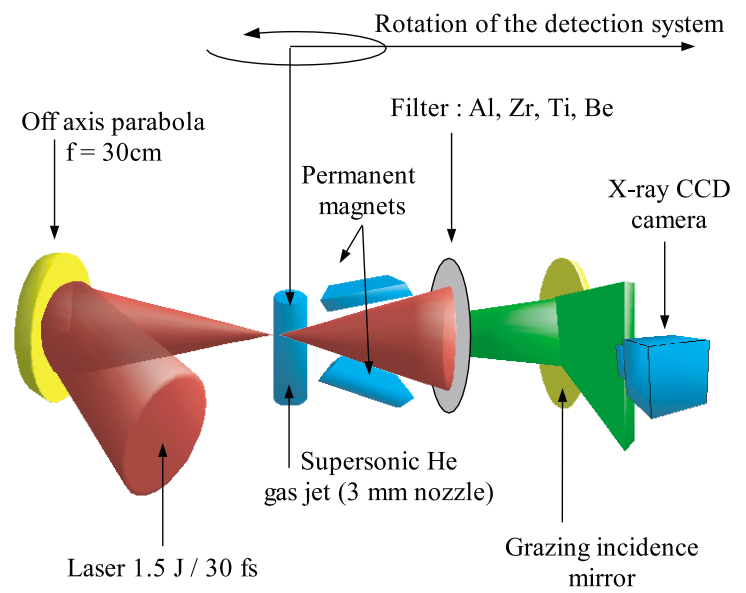

FIG. 1 (color online). Experimental setup. The maximum strength parameter attainable on target is $a_{0}=5.6$.
$100 \mathrm{keV}$ and $100 \mathrm{MeV}$ ranges as well as a magnetic spectrometer positioned in the forward direction [17] in the $10 \mathrm{MeV}$ range.

The behavior of the $\mathrm{x}$-ray intensity as a function of the electron density $\left(n_{e}\right)$ of the plasma as well as the observed $\mathrm{x}$-ray spectral and spatial distributions are presented in Fig. 2 for a laser intensity corresponding to $a_{0}=5$.6. The spatial distribution and the dependency on the electron density are shown for a spectral bandwidth centered on $350 \mathrm{eV}$, but similar results are observed at all the $\mathrm{x}$-ray wavelengths. The observed $x$-ray signal is found to increase linearly with the electron density, to be broad and peaked in the bandwidth centered at $150 \mathrm{eV}$, anisotropic
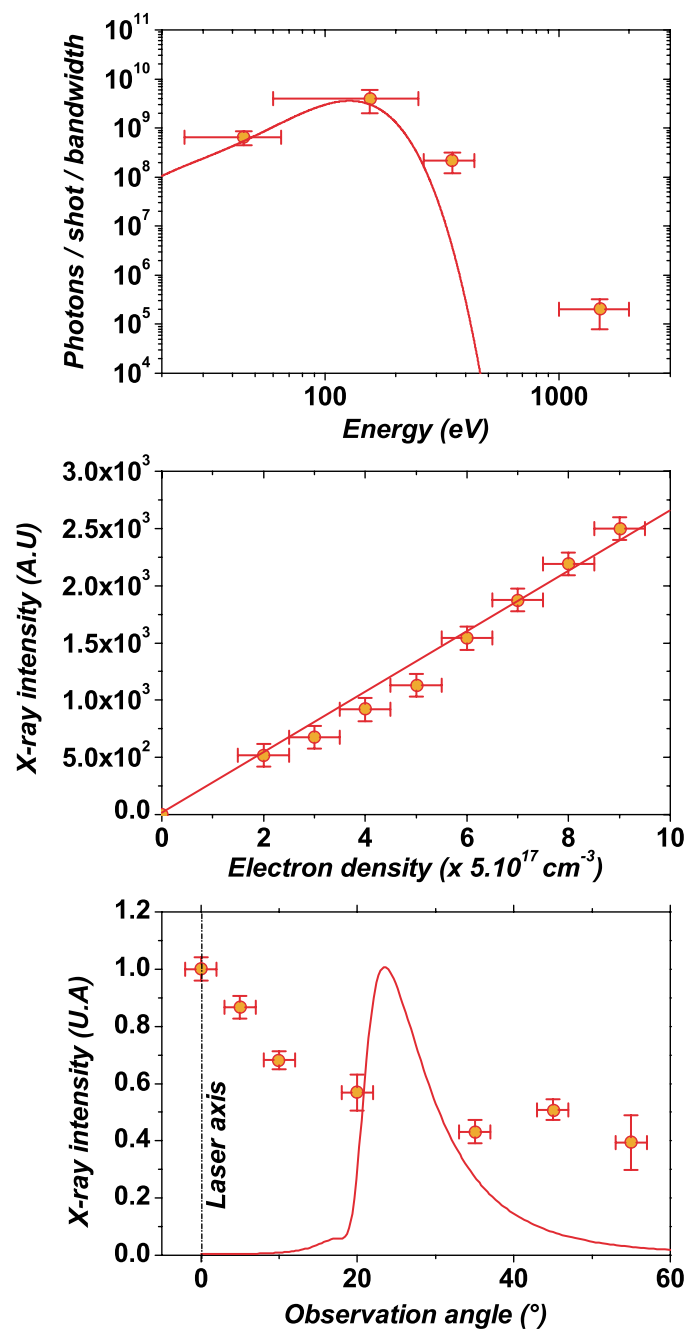

FIG. 2 (color online). (a) Energy spectrum of the observed $\mathrm{x}$-ray emission. The horizontal bars correspond to each spectral bandwidth of the $\mathrm{x}$-ray spectrometer. The vertical error bars correspond to the statistical uncertainty accumulated over five shots. The electron density is $5 \times 10^{19} \mathrm{~cm}^{-3}$ and the angle of observation is $\theta=0^{\circ}$. (b) X-ray intensity as a function of the electron density of the plasma. (c) Angular distribution. The laser beam is focused on the front edge of the gas jet. Solid curves: free electron model depicted in the text. 
and fairly collimated in the forward direction. Such features are consistent with the expected three unique properties of the nonlinear Thomson scattering radiation mentioned previously. However, the simplest model of a free electron initially at rest appears to be insufficient. First, the significant tail up to $2 \mathrm{keV}$ of the spectral distribution obtained on-axis is not expected. The simulations predict a cutoff close to $0.5 \mathrm{keV}$ at the laser intensities achievable with our experimental setup as shown in Fig. 2(a). This signal was very sensitive to the laser propagation and to the position of the focal spot relative to the front edge of the gas jet. Furthermore, electron densities greater than $10^{19} \mathrm{~cm}^{-3}$ are required to observe this higher part of the spectrum. In our experiment, the laser power exceeded the critical power $\left(P_{c}=17 n_{c} / n_{e}\right)$ for relativistic self-focusing of the laser beam inside the plasma by more than 1 order of magnitude. Time-resolved shadowgraphy and side Thomson scattering images of the plasma confirmed the presence of self-guiding. In this regime, the size of the beam is expected to become much smaller than the diameter of the focal spot [18]. The following increase of the parameter $a_{0}(>10)$ may explain the origin of the observed radiation in the $2 \mathrm{keV}$ spectral range.

Second, the angular distribution of the x-ray intensity displayed in Fig. 2(c) for the largest angles clearly shows a residual signal that no longer depends on the angle of observation (the distribution becomes flat from $35^{\circ}$ to $55^{\circ}$ ) indicating that another mechanism becomes responsible for the $\mathrm{x}$-ray emission. To identify this process, the $\mathrm{x}$-ray intensity observed at such large angles of observation has been studied as a function of the electron density and at the same laser intensity as in the experiments presented in Fig. 2(c) $\left(a_{0}=5.6\right)$. The results displayed in Fig. 3 show that a quadratic dependency is found for $\theta=40^{\circ}$ where the spatial distribution is isotropic, indicating that a collisional radiative process (Bremsstrahlung and radiative recombination) from the thermal plasma becomes important. This thermal emission remains less intense than the collimated nonlinear Thomson scattering emission (Fig. 3). It becomes detectable only at a large angle of observation $\left(>40^{\circ}\right)$ and for $a_{0}<1$ (inset of Fig. 3). As expected from the theory, when $a_{0}<1$, the nonlinear Thomson scattering vanishes and the collisional radiative processes prevail. Additional analyses were done to further demonstrate that other radiative processes are not at the origin of the collimated $\mathrm{x}$-ray radiation observed on-axis at high peak laser intensities $\left(a_{0}=5.6\right)$. Tests were done in argon at the same electron density as in helium. No change in the x-ray flux was obtained, whereas a collisional-type emission would have scaled as the square of the atomic number. Furthermore, spatial and time-dependent simulations that take into account these processes show that the emission is still growing with $n_{e}^{2}$. They cannot explain the linear dependency observed on axis. Other processes may participate.

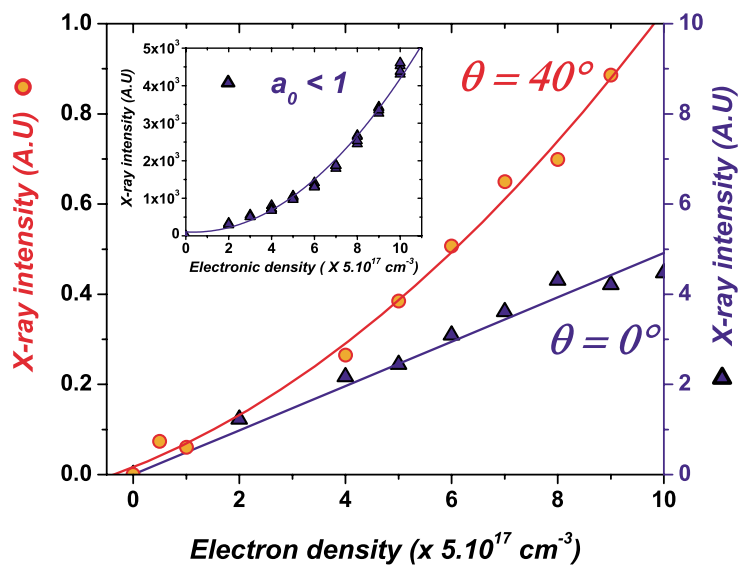

FIG. 3 (color online). X-ray intensity as a function of the electron density of the plasma for $a_{0}=5.6$ and at an angle of observation $\theta=0^{\circ}$ and $40^{\circ}$. The spectral bandwidth is centered at $40 \mathrm{eV}$, the less energetic part of the spectrum. The laser beam is focused on the front edge of the gas jet. The inset shows the $\mathrm{x}$-ray intensity as a function of the electron density of the plasma at an angle of observation $\theta=0^{\circ}$ and for $a_{0}<1$.

Relativistic bremsstrahlung is found to be orders of magnitude smaller than the observed $x$-ray flux [11]. High harmonics from bound electrons could be produced in the wings of the laser pulse where $a_{0}<1$. Experiments done in circular polarization show a significant increase of the $\mathrm{x}$ ray the signal compared to linearly polarized light for all the angles of observations which safely rules out the possibility that this emission from a bound electron occurs [4] in our experimental conditions. Atomic lines from the high temperature plasma generated in the interaction region is isotropic and could also contribute to the uncollimated $x$-ray emission. However, it must be taken into account only for sufficiently high $Z$ elements. In the case of helium, the electron binding energy is limited to $25 \mathrm{eV}$ which is less energetic than the observed radiation spectrum.

Third, the last difference between the observed nonlinear Thomson scattering signal and what was expected from the simple free electron model concerns the spatial distribution. It is found to be much broader than the theoretical width $\left(10^{\circ}\right)$ and to be centered close to the laser axis $\left(\theta=0^{\circ}\right)$ instead of $23^{\circ}$. Similar results were obtained at all the $\mathrm{x}$-ray spectral bandwidths. The characteristic angle of emission is determined by the electron momenta $p_{\perp}$ and $p_{x}$. Those two momenta can be significantly altered if the electrons experience additional acceleration. Electrons accelerated in the forward direction have been clearly measured with radiochromic films and copper filters. Although the set of filters did not allow the characterization of the distribution function, the measurements indicate a strong population of electrons with energy between a few hundred $\mathrm{keV}$ and a few $\mathrm{MeV}$. This population of electrons, which was also seen using a particle in cell simulation, can come from either the 


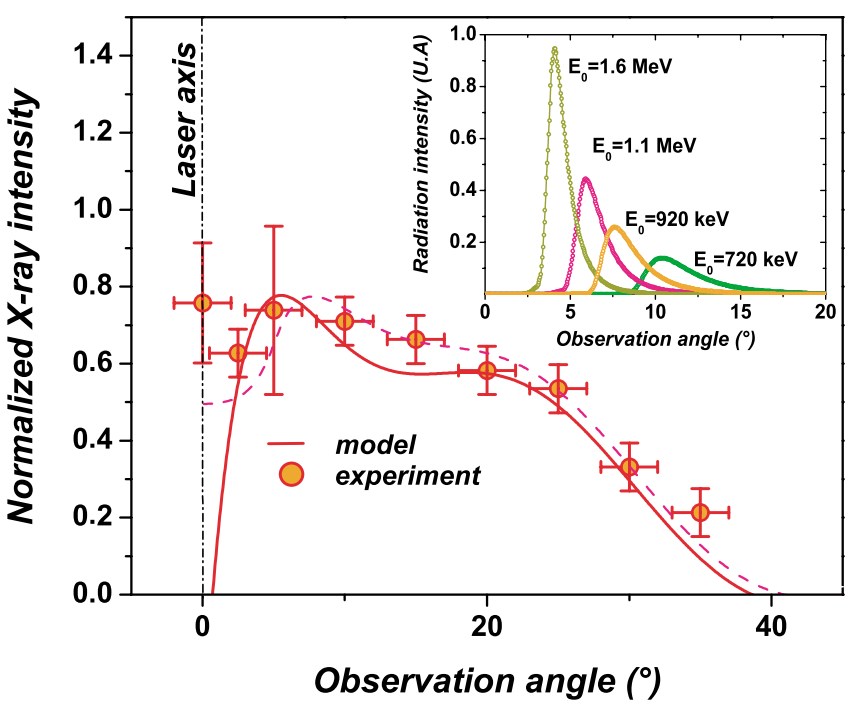

FIG. 4 (color online). Spatial distribution of the observed $\mathrm{x}$-ray emission for $a_{0}=5.6$. The solid line (dotted line) is the numerical result obtained for a Gaussian electron distribution with a temperature of $0.9 \mathrm{MeV}$ and without (with) $5^{\circ} \vec{k}$ spread. The inset shows the effect of the initial energy of monoenergetic electrons on the spatial distribution of the nonlinear Thomson scattering.

wakefield acceleration or a phase mismatch of the electrons inside the laser field due to collisions in the plasma. The consequence is equivalent to a longitudinal energy which comes in addition to the energy acquired in the electromagnetic field and the ratio $p_{x} / p_{\perp}$ can be strongly increased. Numerical simulations were done to estimate this effect on the spatial distribution (inset of Fig. 4) [19]. We have found that monoenergetic electrons at $0.7 \mathrm{MeV}$ produce an emission centered on $\theta=10^{\circ}$ instead of $23^{\circ}$ for free electrons. Electrons at $1.6 \mathrm{MeV}$ further collimate the radiation to $4^{\circ}$. An electron distribution function, as seen in this experiment, can clearly broaden the $\mathrm{x}$-ray signal and center it towards the axis. The best fit of the experimental data is shown in Fig. 4 by also taking into account the contribution of a $5^{\circ} \vec{k}$ spread which can come from either the divergence of the electron beam or the spread of the $k$ vector of the laser. An electron distribution function $\exp \left(-E / E_{0}\right)$ characterized by an electronic temperature of $0.9 \mathrm{MeV} \pm 0.3 \mathrm{MeV}$ is obtained, which is found to be in the range of energies detected experimentally.

In conclusion, we have demonstrated that the observed $\mathrm{x}$-ray signal follows the unique features of the nonlinear Thomson scattering radiation: angular distribution, spectral distribution, as well as the scaling with the electron density. The simple picture of the free electron oscillating inside the laser field is not sufficient to describe the electron-photon interaction. Additional effects such as plasma-accelerated electrons must be taken into account and result in a shift and a broadening of the characteristic angle of emission. We have estimated the number of $x$-ray photons, integrated over the angular distribution and the spectrum, to be $5 \times 10^{10}$ per shot which is in close agreement with the prediction from the nonlinear Thomson scattering theory $\left(10^{11}\right)$. The duration of the $\mathrm{x}$-ray pulse is mainly controlled by that of the laser. However, the speed of the laser propagating inside the plasma is slightly below the speed of light and a temporal mismatch between the $\mathrm{x}$ rays (traveling at the speed of light) could occur. This mismatch is estimated to be about $10 \mathrm{fs}$ when the larger size of the plasma is being used $(3 \mathrm{~mm})$. The temporal stretching of the $\mathrm{x}$-ray pulse duration is not significant compared to the duration of the laser and a 30 fs polychromatic x-ray burst is likely to have been produced. The measurement of the $\mathrm{x}$-ray pulse duration would be an additional confirmation that the $\mathrm{x}$-ray emission is produced by nonlinear Thomson scattering.

This work was supported by the European Community under Contracts No. HPRI-CT-1999-00086, No. HPRICT-2000-40016, and No. HPRI-CT-1999-50004. D. U. was supported by DOE, Division of Chemical Physics Office of Science. We thank E. Lefebvre from CEA for fruitful discussions.

*Corresponding author.

Electronic address: antoine.rousse@ensta.fr

[1] A. Rousse, C. Rischel, and J. C. Gauthier, Rev. Mod. Phys.. 73, 17 (2001).

[2] C. Rischel et al., Nature (London) 390, 490 (1997).

[3] A. Rousse et al., Nature (London) 410, 65 (2001).

[4] T. Brabec and F. Krausz, Rev. Mod. Phys. 72, 545 (2000).

[5] M. Murnane et al., Science 251, 531 (1991).

[6] A. Rousse et al., Phys. Rev. E 50, 2200 (1994).

[7] E. Esarey, S. K. Ride, and P. Sprangle, Phys. Rev. E 48, 3003 (1993).

[8] S. K. Ride et al., Phys. Rev. E 52, 5425 (1995).

[9] E. S. Sarachik and G. T. Shappert, Phys. Rev. D 1, 2738 (1970).

[10] B. Shen et al., Opt. Commun. 136, 239-242 (1997).

[11] Y. Ueshima et al., Laser Part. Beams 17, 45 (1999).

[12] K. J. Kim et al., Nucl. Instrum. Methods Phys. Res., Sect. A 341, 351-354 (1994).

[13] W. P. Leemans et al., IEEE J. Quantum Electron. 33, 1925-1933 (1997).

[14] S. Y. Chen, A. Maksimchuk, and D. Umstadter, Nature (London) 396, 653 (1998).

[15] S. Banerjee et al., Phys. Plasmas 9, 2393-2398 (2002).

[16] J.D. Jackson, Classical Electrodynamics (John Wiley and Sons, Inc., New York, 1998), 3rd ed.

[17] V. Malka et al., Phys. Plasmas 8, 2605 (2001).

[18] A. Pukhov and J. Meyer-ter-Vehn, Phys. Rev. Lett. 76, 3975 (1996).

[19] Y. Salamin and F. H. M Faisal, Phys. Rev. A 54, 4383 (1996). 